\title{
REVIEW
}

\section{Wnt5a Plays Controversial Roles in Cancer Progression}

\author{
Xu Chen ${ }^{1}$, Hongling Liư ${ }^{2}$, Fenghui Zhao ${ }^{1 *}$, \\ Zongxian Jiao $^{3}$, Jinsui Wang ${ }^{1}$, Yamei Dang ${ }^{1}$ \\ 1 Department of Pathology, Gansu Provincial \\ Hospital, Lanzhou 730000, China \\ ${ }^{2}$ Department of Occupational Medicine, the Third Gansu \\ Provincial Hospital, Lanzhou 730000, China \\ ${ }^{3}$ Pathological Institution of Basic Medical College, Lanzhou \\ University, Lanzhou 730000, China
}

Key words: Wnt5a; cancer; methyl modification

\begin{abstract}
Wnt5a is a representative Wnt ligand that regulates multiple cellular functions through the Wnt5a nonclassical pathway. Although Wnt5a has been implicated in various pathological conditions, its role in cancer is ambiguous and might involve methyl modifications, distinct mRNA isoforms, as well as different downstream pathways. Therefore, it is an essential factor in cancers' progression (invasion, migration, proliferation, and epithelial-mesenchymal transition), and a potential biomarker for prognosis and treatment.
\end{abstract}

W

NT factors regulate various cellular functions, including proliferation, migration and differentiation. ${ }^{[1,2]}$ They encompass over 19 members in mice and humans, and are modified by cysteine-rich secretory ligands. ${ }^{[3]}$ Based on the downstream signaling pathways, Wnt factors are classified into the $\beta$-catenin-dependent (e.g., Wnt1, Wnt3, Wnt3a and Wnt7a) and $\beta$-catenin-independent (e.g., Wnt2, Wnt4, Wnt5a, Wnt5b, Wnt6, Wnt7b and Wnt11) pathway activators. ${ }^{[4]}$ Wnt5a is highly expressed during embryonic development,

Received September 12, 2019; accepted February 4, 2020; published online September 27, 2020.

*Corresponding author E-mail: zhaofh0931@163.com.

Fund supported by the Gansu Health and Family Planning

Commission Funding (GSWSKY2018-34). and declines thereafter in normal adult tissues. ${ }^{[5,6]}$ Aberrant Wnt5a expression levels have been reported in various cancers, wherein it plays contrasting roles. ${ }^{[7,8]}$ For example, its short isoform (Wnt5a-S) containing 319 amino acids promotes cancer cell proliferation, while its longer isoform (Wnt5a-L) with 337 amino acids has an inhibitory effect and it is regulated by hypermethylation of WNT5A promotor region. ${ }^{[9]}$ In this review, the role of Wnt5a in cancers and the associated mechanisms have been discussed.

\section{WNT5A AND ITS RECEPTORS}

The Wnt5a gene was first identified by Gavin et $a^{\left[{ }^{[10]}\right.}$ and Nusse \& Varmus ${ }^{[11]}$ in mice, and mapped to chromosome 14. Subsequently, Clark et al. ${ }^{[12]}$ identified the human Wnt5a gene on chromosome 3 
(3p14-p21). Wnt5a is a glycosylated protein that contains conserved cysteine (Cys77 and Cys104) ${ }^{[12]}$ and serine residues (Ser209 and Ser244) that undergo palmitoylation, i.e., covalent attachment to palmitate moieties, before binding to its frizzled receptors. ${ }^{[13]}$ Ten frizzled (Fzs) seven-pass transmembrane proteins have been identified in mammals so far. Wnt5a binds to Fz3, Fz4, Fz5 and Fz8, and induces DSHI (disheveled 1) phosphorylation. ${ }^{[14]}$ In addition, binding to Fz7 triggers activating protein 1 (AP-1) activation and Dishevelled (Dvl) polymerization, and that to Fz2 increases intracellular calcium concentration and activates Rac. ${ }^{[15]}$ Thus, Wnt5a can regulate multiple cellular functions via Fzs binding.

Tyrosine kinase-like orphan receptor 2 (Ror2), a single-pass transmembrane protein with a tyrosine kinase domain, is an essential receptor of Wnt5a. ${ }^{[16]}$ The latter binds to Ror2 forming a ternary complex, which then interacts with Fzs and activates Wnt5a signaling. The transmembrane receptor-like tyrosine kinase (Ryk) is another essential Wnt5a receptor, ${ }^{[17]}$ which binds to Wnt5a and other Wnts. ${ }^{[18]}$ Some Wnts, like Wnt1a and Wnt3a, can bind to Fzs and low-density lipoprotein receptor-related protein co-receptor 5/6 (LRP5/6), initiating canonical Wnt signals. ${ }^{[19,20]}$ In addition, Wnt-receptor binding inhibits the adenomatous polyposis coli (APC) and glycogen synthase kinase $3 \beta$ (GSK3 $\beta$ ) complex, which frees the cytoplasmic $\beta$-catenin that then binds to T-cell factor (TCF)/lymphoid enhancer factor (LEF), and activates the transcription of Wnt target genes. ${ }^{[21,22]}$ However, Wnt5a also promotes $\beta$-catenin degradation, thus inhibites $\beta$-catenin-TCF/LEF binding and canonical Wnt genes transcription. ${ }^{[22]}$ (Figure 1)

Wnt5a regulates convergent extension movements by stimulating the $\mathrm{Ca}^{2+}$ signaling pathway, ${ }^{[23]}$ wherein the Wnt5a-Fzs complex activates recruitment of disheveled (DSH) proteins and phospholipase C (PLC), and mediates intracellular $\mathrm{Ca}^{2+}$ release. ${ }^{[24-26]}$ The increased $\mathrm{Ca}^{2+}$ levels activate calmodulin dependent protein kinase II (CAMK II ) or protein kinase C (PKC), leading to de-phosphorylation of nuclear factor of activated T cell (NFAT). ${ }^{[27,28]}$ This pathway is crucial in regulating cell movement, proliferation and migration. ${ }^{[28]}$ Wnt5a also regulates cellular gastrulation and maintenance via the planar cell polarity (PCP) pathway. ${ }^{[29]}$ The Wnt5a-Ror2 complex can directly stimulate Jun $\mathrm{N}$-terminal kinase (JNK), leading to uniform polarization, ${ }^{[30]}$ as well as indirectly activate DSH and then the small GTPases Rho and Rac. ${ }^{[31,32]}$ (Figure 1)

\section{WNT5A AND CANCER}

Studies increasingly show different roles of Wnt5a in various cancers. Wnt5a-mediated non-classical pathway can antagonize the functions of the canonical Wnt/ $\beta$-catenin pathway and inhibit cancer progression. ${ }^{[33,34]}$ It also activates CAMK $\mathbb{I}_{,}^{[35]} \operatorname{siah} 2^{[36]}$ or ROR2 ${ }^{[37]}$ to promote $\beta$-catenin degradation, and activates interleukin (IL)-10 upon Fzs binding to inhibit the TLR4-NF-kB signaling pathway. ${ }^{[38]}$ (Figure 2A) On the other hand, the Wnt5a/Ror2 signaling pathway can also promote cancer progression ${ }^{[39]}$ via the pro-tumorigenic PI3K and PKC pathways. ${ }^{[40]}$ Furthermore, Wnt5a triggers lamellipodia and filopodia formation, thus promoting cancer metastasis and invasion. ${ }^{[41]}$ (Figure 2B)

\section{Gastric cancer and colorectal cancer}

Wnt5a overexpression is observed in diffusive-type gastric cancer, ${ }^{[42,43]}$ and is associated with tumor cell migration and invasion. It promotes in vitro migration of the gastric cancer SGC-7901 cell line via the GS3K $/$ / PI3K/AKT pathway, ${ }^{[44]}$ while antibody-mediated inhibition of Wnt5a suppresses the migration and invasion of the MKN-1, KKLS and TMK-1 cell lines. ${ }^{[45]}$ Wnt5a overexpression promotes growth of KKLS and TMK-1 cells via laminin $\mathrm{Y}_{1}{ }^{[46]}$ induces Snail overexpression and upregulates CD113 in MKN-7 cells, ${ }^{[4]}$ triggers epithelial-mesenchymal transition (EMT) in the SGC-7901 cells via Arf6ERK signaling, ${ }^{[48]}$ and promotes proliferation of MNK-45 cells by activating CXCL16-CXCR6 signaling. ${ }^{[49]}$ Taken together, Wnt5a acts as an oncogene in gastric cancer and drives tumor progression. In contrast, Wnt5a overexpression indicates good prognosis in Dukes B colorectal cancer (CRC) ${ }^{\left[{ }^{[50]}\right.}$ In addition, the highly metastatic CRC cell line SW620 shows low level of Wnt5a, which is increased upon inhibition of EMT and metastasis. ${ }^{[1]}$ CRC patients with Wnt5a overexpress in the tumor tissues survive longer compared to those with Wnt5a loss, which may be linked to Wnt5a-mediated upregulation of 15-hydroxyprostaglandin dehydrogenase (15-PGDH). ${ }^{[52]}$ Wnt5a also inhibits proliferation of the HCT116 and SW480 cell lines, ${ }^{[53]}$ and is downregulated in the latter when EMT is stimulated by TGF- $\beta{ }^{\left[{ }^{[54]}\right.}$ However, one study showed that Wnt5a promoted CRC progression in the Apc1638N mouse model by stimulating cell proliferation and invasion. ${ }^{[55]}$ These opposite effects might involve different WNT5A mRNA isoforms. For instance, Wnt5a-L mRNA inhibits proliferation of the HCT116 cells, whereas Wnt5a-S mRNA has a pro-growth effect. ${ }^{[56]}$ 


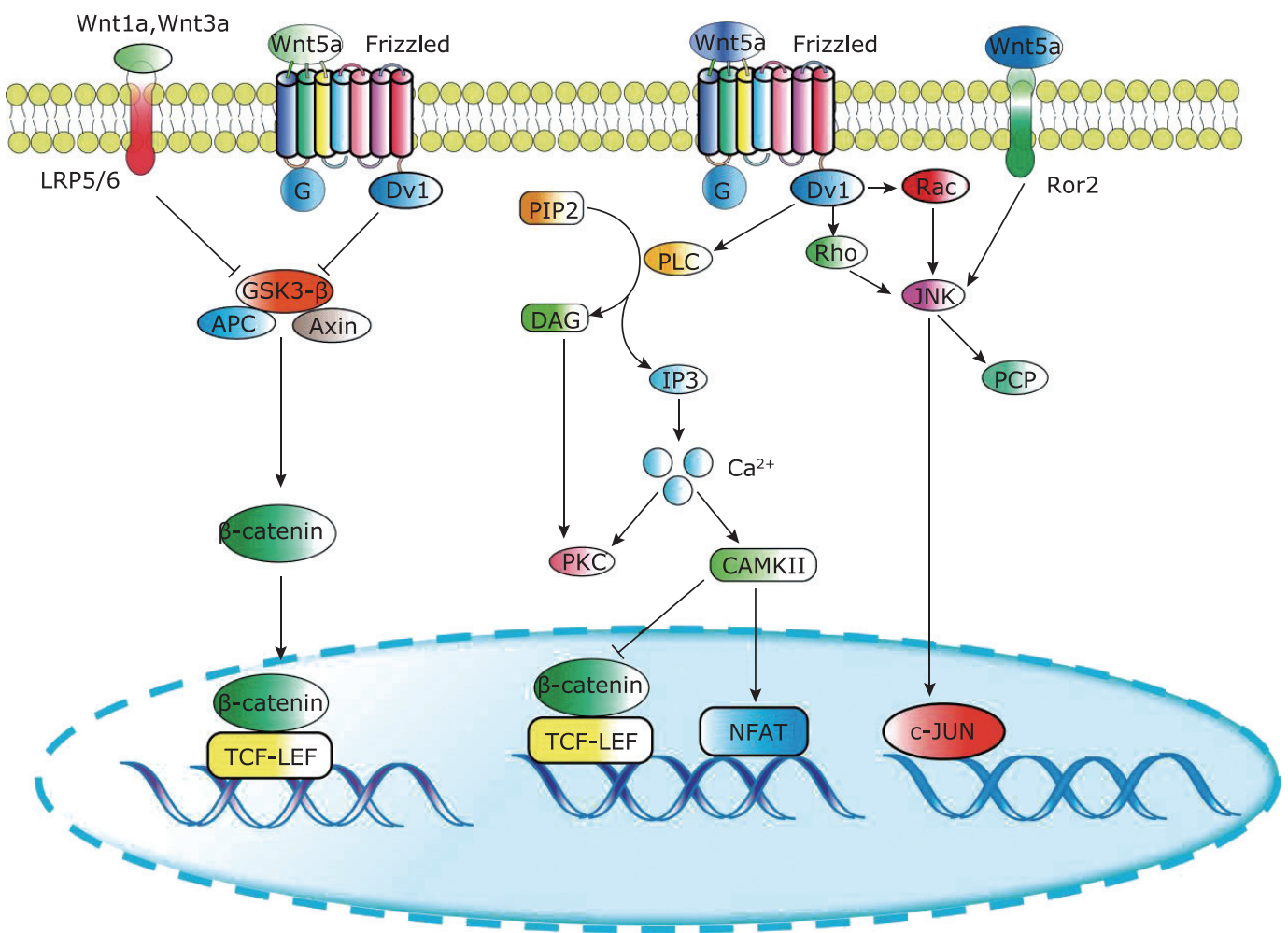

Figure 1. Wnt signaling pathway. The two groups of Wnt factors regulate the $\beta$-catenin, calcium and planar cell polarity $(\mathrm{PCP})$ /convergent extension (CE) pathways.

APC, adenomatous polyposis coli; CAMK II , calmodulin dependent protein kinase II ; LRP, lipoprotein receptor-related protein co-receptor; DAG, diacylglycerol; Dvl, dishevelled; IP3, inositol 1,4,5-trisphosphate; JNK, Jun N-terminal kinase; NFAT, nuclear factor of activated T cell; PIP2, phosphatidylinositol 4,5-bisphosphate; PLC, proteins and phospholipase C; PKC, protein kinase C; Ror2, receptor tyrosine kinase-like orphan receptor 2; TCF, T-cell factor; LEF, lymphoid enhancer factor.

A

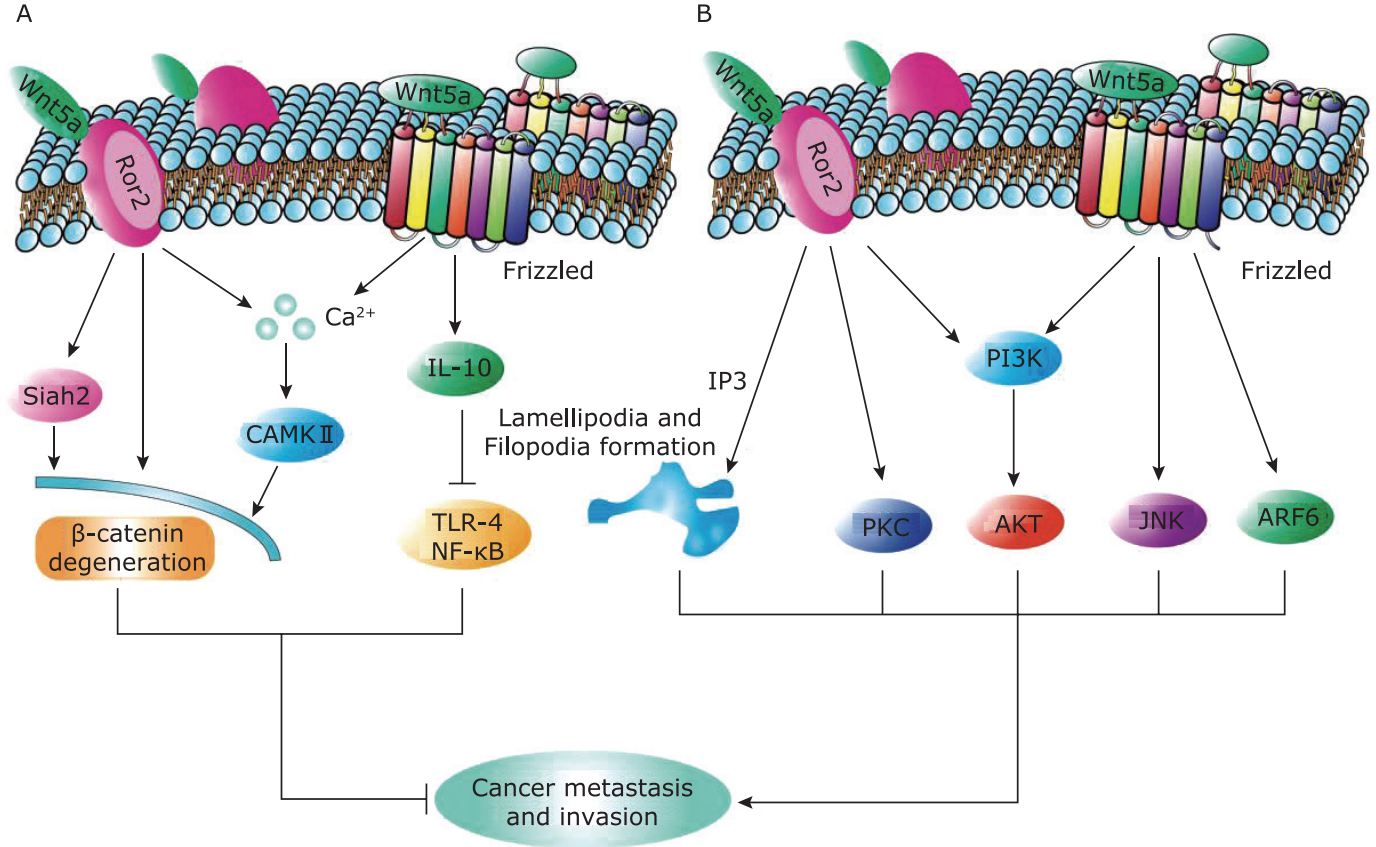

Figure 2. Ambiguous roles of Wnt5a in cancer metastasis.

(A) Inhibition of metastasis in cancer. (B) Promotion of metastasis in cancer. ARF6, ADP-ribosylation factor 6; NF-kB, nuclear factor-kB; IL-10, interleukin 10; PI3K, phosphatidylinositol 3 - kinase; Siah2, absentia homologues 2; TLR-4, toll-like receptor-4. 


\section{Non-small-cell lung cancer and breast cancer}

Non-small-cell lung cancer (NSCLC) is a highly aggressive, metastatic disease with poor prognosis, ${ }^{[57]}$ and Wnt5a overexpression has been observed in NSCLC patients. ${ }^{[58]}$ Wnt5a down-regulation inhibits NSCLC cell invasion, migration and $\mathrm{EMT}^{[59]}$ while its up-regulation induced migration and clone formation of the $\mathrm{H} 1975$ cell line. ${ }^{[59]}$ In NSCLC patients, Wnt5a expression is positively associated with that of stromal vascular endothelial growth factor, and might improve tumor neo-angiogenesis. ${ }^{[60]}$ Wnt5a promotes malignant clone formation in cigarette smoking-related NSCLC, ${ }^{[61]}$ and induces cisplatin-resistance in A549 cells by activating the PKC signaling pathway. ${ }^{[62]}$

The role of Wnt5a in breast cancer is more ambiguous. While some studies showed an inhibitory effect of Wnt5a, ${ }^{[63]}$ and its overexpression in estrogen-receptor (ER) positive breast cancer patients indicated good prognosis. ${ }^{[63]}$ Other studies have reported lower levels of Wnt5a in breast cancer tissues compared to normal tissues. ${ }^{[64]}$ In addition, Wnt5a also inhibits migration and proliferation of the MDA-MB-231 and MDA-MB-468 cell lines by various mechanisms such as activating $\mathrm{Cdc} 42,{ }^{[65,66]}$ reducing phosphofructokinase platelet-type (PFKP) expression, ${ }^{[33]}$ splicing CD44 Mrna, ${ }^{[67]}$ and downregulating CD44 expression levels. ${ }^{[64]}$ However, there are reports of Wnt5a promoting invasion of the MDA-MB-231 cells by activating NF-KB and upregulating MMP-7 expression. ${ }^{[68]}$ Furthermore, down-regulation of Wnt5a in the MDA-MB-175-VII cells inhibits their migration while Wnt5a overexpression increases malignancy of ER-positive breast cancer. ${ }^{[63]}$ Finally, it induces the migration in MDA-MB-231 and MCF-7 cells by stimulating the Dvl2/ Daam1/RhoA pathway. ${ }^{[69]}$ In conclusion, Wnt5a has an overall pro-tumorigenic role in NSCLC and breast cancer, although the mechanisms need to be elucidated.

\section{Pancreatic cancer and prostate cancer}

Wnt5a overexpression in pancreatic cancer patients indicates poor prognosis and lymph node metastasis, ${ }^{[70]}$ and promotes the proliferation and reduces apoptosis in PANC-1 and BXPC-3 cells. ${ }^{[71]}$ In the murine pancreatic tumor model, Wnt5a overexpression induced EMT and cancer cell metastasis. ${ }^{[72]}$ Furthermore, it promotes migration of the PANC1, Capan-2 and HT1080 cells by phosphorylating paxillin, ${ }^{[73]}$ and induces gemcitabine resistance in PANC-1 and MIAPaCa2 cells via Cyclin D1 activation and AKT phosphorylation. ${ }^{[74]}$

Prostate cancer has a high rate of bone metas- tasis. In some patients, Wnt5a overexpression indicated poor prognosis. ${ }^{[75,76]}$ It induces castration resistance in the LNCaP and 22Rv1 cell lines by stimulating BMP-6 $6^{[75,77]}$ and chemokine ligand 2 (CCL2) expression. ${ }^{[78]}$ In the PC3, LNCaP, and DU145 cells, Wnt5a down-regulation can reduce proliferation and migration. ${ }^{[79]}$ However, one study showed that Wnt5a overexpression in PC3, C42B, and MDA-PCa-2b cells inhibited their proliferation and induced apoptosis. ${ }^{[80]}$ Furthermore, localized overexpression of Wnt5a in some prostate cancer patients indicates good prognosis. ${ }^{[81]}$ In conclusion, Wnt5a has an oncogenic role in pancreatic cancer and a more complex function in prostate cancer, which likely depends on the downstream pathway.

\section{Melanoma and leukemia}

Wnt5a promotes melanoma progression, and high level of Wnt5a in melanoma patient is associated with poor prognosis. ${ }^{[82]}$ In the melanoma cell line UACC 1273, Wnt5a overexpression induces in vitro invasion and proliferation by stimulating protein kinase $\mathrm{C}$ expression, ${ }^{[83]}$ while blocking Wnt5a in the HTB63 and A375 cells reduced migration. ${ }^{[83]}$ It also promotes the invasion and migration of the SKmel28, A2058, A375 and HTB63 cells by stimulating exosomes release, ${ }^{[84]}$ and enhances chemo-resistance against BRAF inhibitors in A543 and MEL624 cells by activating AKT. ${ }^{[85]}$ Thus, Wnt5a promotes melanoma progression by inducing cell invasion, migration, proliferation, and chemo-resistance.

In leukemia, the role of Wnt5a is ambiguous. While some studies show that Wnt5a overexpression is a protective factor in acute lymphoblastic leukemia, ${ }^{[86]}$ and Wnt5a silence is observed in acute leukemia; ${ }^{[87]}$ other studies have illustrated that Wnt5a overexpression promotes leukemia cells proliferation and migration via inducing Ror1 and Ror2. ${ }^{[88,89]}$ These opposite effects might involve Wnt5a epigenetic changes. For instance, hypermethylation of WNT5A promotor region is observed in acute lymphoblastic leukemia and it leads to downregulation of WNT5A mRNA level. ${ }^{[90]}$ In acute leukemia, histone H4K20me1 is enriched in WNT5A promotor and coding region, regulating Wnt5a expression via inducing transcription elongation and initiation. ${ }^{[91]}$

\section{CONCLUSION}

Wnt5a has an ambiguous role in tumorigenesis, as indicated by the conflicting reports in different cancer types. Wnt5a can influence on cell proliferation, invasion, 
migration, metastasis, and chemo-resistance. While it promotes tumor progression in gastric cancer, NSCLC, pancreatic cancer and melanoma, Wnt5a has opposite effects in colorectal cancer, breast cancer and prostate cancer, which likely involves distinct signaling pathways. Furthermore, in colorectal cancer and leukemia, distinct WNT5A isoforms and epigenetic modification lead to different roles of Wnt5a. WNT5A DNA hypermethylation and histone methylation might influence on WNT5A mRNA isoforms and Wnt5a protein expression, leading to different Wnt5a expression in cancers. Furthermore, Wnt5a regulates various signal pathways and these pathways have distinct effects on cancer progression (Figure 3 ). In conclusion, WNT5A methyl modification and Wnt5a protein effects on various signal pathways might contribute to its controversial roles in cancers.

\section{Conflict of interests}

All authors declared no conflicting interests.

\section{REFERENCES}

1. Wang $X$, Zhao X, Yi Z, et al. WNT5A promotes migra- tion and invasion of human osteosarcoma cells via SRC/ERK/MMP-14 pathway. Cell Bio Int 2018; 42(5): 598-607. doi: 10.1002/cbin.10936.

2. Wang $W, Y u X$, Wu C, et al. Differential effects of Wnt5a on the proliferation, differentiation and inflammatory response of keratinocytes. Mol Med Rep 2017; 17(3):4043-48. doi: 10.3892/mmr.2017.8358.

3. Feng $C$, Liang $S$, Harold V. Wnt signals across the plasma membrane to activate the beta-catenin pathway by forming oligomers containing its receptors, Frizzled and LRP. Development 2004; 131:5103-15. doi: 10.1089/scd.2015.0367.

4. Zins $K$, Schäfer $R$, Paulus $P$, et al. Frizzled2 signaling regulates growth of high-risk neuroblastomas by interfering with $\beta$-catenin-dependent and $\beta$-catenin-independent signaling pathways. Oncotarget 2016; 7(29):46187-202. doi: 10.18632/oncotarget.10070.

5. Keller KC, Ding H, Tieu R, et al. Wnt5a Supports Osteogenic Lineage Decisions in Embryonic Stem Cells. Stem Cells Dev 2016; 25(13):1020-32. doi: 10.1089/ scd.2015.0367.

6. Abedini A, Zamberlam G, Lapointe E, et al. WNT5a is required for normal ovarian follicle development and

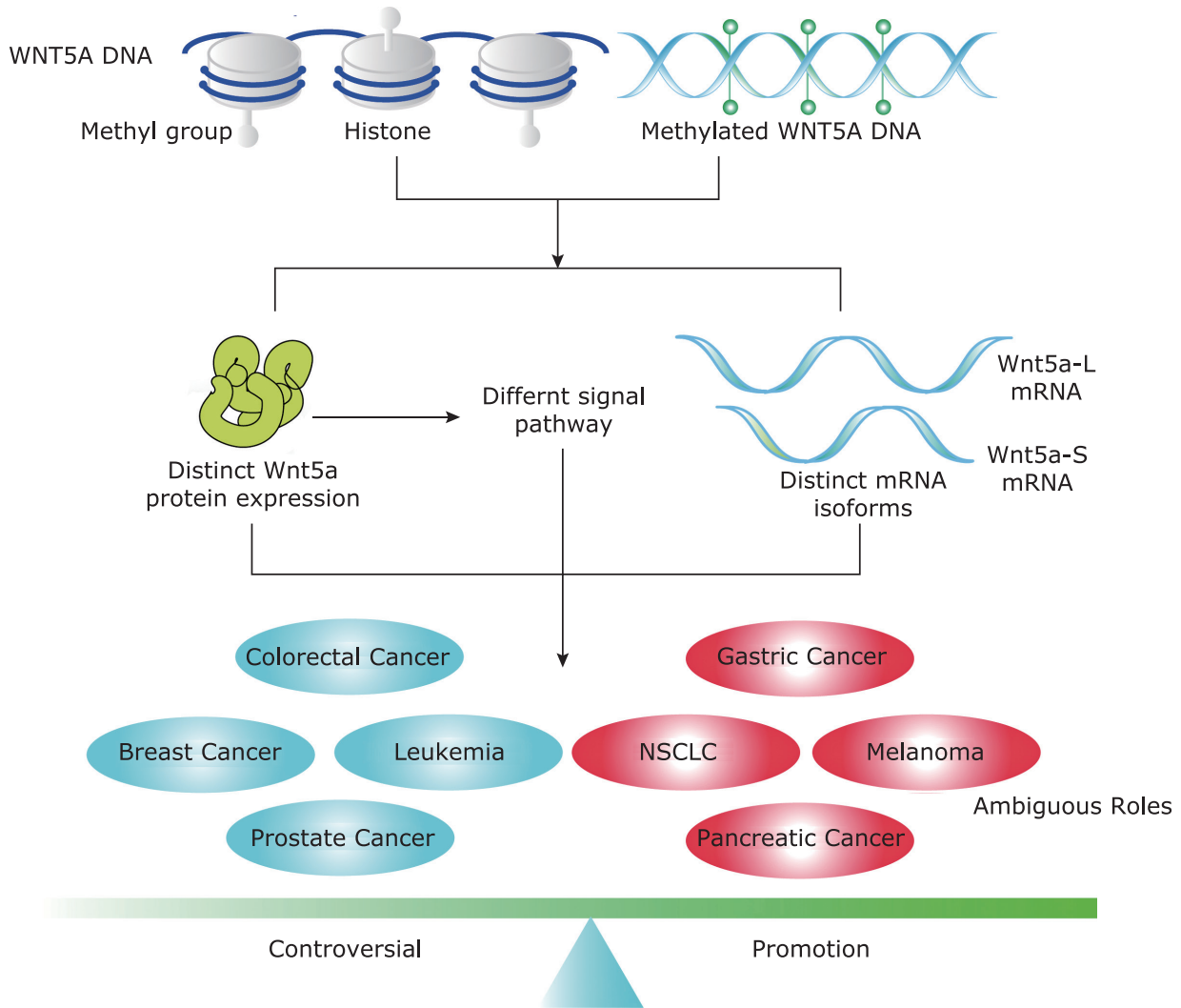

Figure 3. WNT5A methyl modification might influence on Wnt5a controversial roles.

Gene methylation might lead to distinct mRNA isoforms. Furthermore, WNT5A methyl modification influence on Wnt5a expression level and Wnt5a acts on different signal pathway. 
antagonizes gonadotropin responsiveness in granulosa cells by suppressing canonical WNT signaling. Faseb J 2016; 30(4):1534-47. doi: 10.1096/fj.15-280313.

7. Saling M, Duckett JK, Ackers I, et al. Wnt5a/planar cell polarity signaling pathway in urothelial carcinoma, a potential prognostic biomarker. Oncotarget 2017; 8(19):31655-65. doi: 10.18632/oncotarget.15877.

8. Zhong Z, Shan M, Wang J, et al. Decreased Wnt5a Expression is a Poor Prognostic Factor in Triple-Negative Breast Cancer. Med Sci Monit 2016; 22:1-7. doi: 10.12659/MSM.894821

9. Matthieu B, Jean B, Terry G, et al. WNT5A encodes two isoforms with distinct functions in cancers. Plos One 2013; 8:e80526. doi: 10.1371/journal.pone. 0080526.

10. Gavin BJ, Mcmahon JA, Mcmahon AP. Expression of multiple novel Wnt-1/int-1-related genes during fetal and adult mouse development. Genes Dev 1990; 4(12B):2319-32. doi: 10.1101/gad.4.12b.2319.

11. Parr BA, Mcmahon AP. Wnt genes and vertebrate development. Cur Opinion Genet Develop 1994; 4(4):523-8. doi: 10.1016/0959-437X(94)90067-D.

12. Clark CC, Cohen I, Eichstetter I, et al. Molecular cloning of the human proto-oncogene Wnt-5A and mapping of the gene (WNT5A) to chromosome 3p14-p21. Genomics 1993; 18(2):249-60. doi: 10.1006/ geno.1993.1463.

13. Lin HK, Lin HH, Chiou YW, et al. Caveolin-1 down-regulation is required for Wnt5a-Frizzled 2 signalling in Ha-RasV12-induced cell transformation. J Cellular Molecular Med 2018; 22(5):2631-43. doi: 10.1111/ jcmm.13531.

14. Wang HY, Liu T, Malbon CC. Structure-function analysis of Frizzleds. Cell Signal 2006; 18(7):934-41. doi: 10.1016/j.cellsig.2005.12.008.

15. Bischoff DS, Zhu JH, Makhijani NS, et al. Induction of CXC chemokines in human mesenchymal stem cells by stimulation with secreted frizzled-related proteins through non-canonical Wht signaling. World J Stem Cells 2015; 7(11):1262-73. doi: 10.4252/wjsc. v7.i11.1262.

16. Uehara S, Udagawa N, Mukai $H$, et al. Protein kinase $\mathrm{N} 3$ promotes bone resorption by osteoclasts in response to Wnt5a-Ror2 signaling. Sci Signaling 2017; 10(494):e0023. doi: 10.1126/scisignal.aan0023.

17. Skaria T, Bachli E, Schoedon G. Wnt5A/Ryk signaling critically affects barrier function in human vascular endothelial cells. Cell Adh Migr 2016; 11(1):24-38. doi: $10.1080 / 19336918.2016 .1178449$.
18. Adamo A, Fiore D, Martino FD, et al. RYK promotes the stemness of glioblastoma cells via the WNT/ $\beta$-catenin pathway. Oncotarget 2017; 8(26):1347687. doi: 10.18632/oncotarget. 14564.

19. Sakisaka $Y$, Kanaya $S$, Nakamura $T$, et al. p38 MAP kinase is required for Wnt3a-mediated osterix expression independently of Wnt-LRP5/6-GSK3 $\beta$ signaling axis in dental follicle cells. Biochem Biophys Res Commun 2016; 478(2):527-32. doi: 10.1016/ j.bbrc.2016.07.076.

20. Lai K, Kweon SM, Chi F, et al. Abstract 4331: Novel Wnt-SCD-LRP5/6 pathway linking liver fibrosis to cancer. Cancer Res 2017; 77. doi: 10.1158/1538-7445. AM2017-4331.

21. Kim JG, Kim MJ, Choi WJ, et al. Wnt3A Induces GSK$3 \beta$ Phosphorylation and $\beta$-Catenin Accumulation Through RhoA/ROCK. J cell physiol 2017; 232(5). 1104-13. doi: 10.1002/jcp. 25572.

22. Daniels $D L$, Weis WI. $\beta$-catenin directly displaces Groucho/TLE repressors from Tcf/Lef in Wnt-mediated transcription activation. Nat Struct Mol Biol 2005; 12(4):364-71. doi: 10.1038/nsmb912.

23. Yu L, Liu S, Zhao Z, et al. Extracorporeal shock wave rebuilt subchondral bone in vivo and activated $\mathrm{Wn}$ $\mathrm{t} 5 \mathrm{a} / \mathrm{Ca}^{2+}$ signaling in vitro. Biomed Res Int 2017; 2017:1404650. doi: 10.1155/2017/1404650.

24. Hatziapostolou M, Koukos G, Polytarchou C, et al. Tumor progression locus 2 mediates signal-induced increases in cytoplasmic calcium and cell migration. Sci Signal 2011; 4(187):e55. doi: 10.1126/scisignal. 2002006.

25. Ho HYH, Susman MW, Bikoff JB, et al. Wnt5a-Ror-Dishevelled signaling constitutes a core developmental pathway that controls tissue morphogenesis. Proc Natl Acad Sci U S A 2012; 109(1):4044-51. doi: 10.1073/ pnas. 1200421109

26. Martineau X, Abed É, Martel-Pelletier J, et al. Alteration of Wnt5a expression and of the non-canonical Wnt/PCP and Wnt/PKC-Ca ${ }^{2+}$ pathways in human osteoarthritis osteoblasts. Plos One 2017; 12(8):e0180711. doi: 10.1371/journal.pone.0180711.

27. Chen $M$, Zhong $W$, Hu $Y$, et al. Wnt5a/FZD5/CaMKII signaling pathway mediates the effect of BML-111 on inflammatory reactions in sepsis. Int J Clin Exp Med 2015; 8(10):17824-9.

28. Chattopadhyay S, Chatterjee R, Law S. Noncanonical Wnt5a-Ca ${ }^{2+}$-NFAT signaling axis in pesticide induced bone marrow aplasia mouse model: A study to explore the novel mechanism of pesticide toxicity. 
Environ Toxicol 2016(10); 31:1163-75. doi: 10.1002/ tox.22123.

29. Saling M, Duckett JK, Ackers I, et al. Wnt5a/planar cell polarity signaling pathway in urothelial carcinoma, a potential prognostic biomarker. Oncotarget 2017; 8(19):31655-65. doi: 10.18632/oncotarget.15877.

30. Hasegawa D, Wada N, Yoshida S, et al. Wnt5a suppresses osteoblastic differentiation of human periodontal ligament stem cell-like cells via Ror2/JNK signaling. J cell physiol 2018; 233(2):1752-62. doi: $10.1002 / j c p .26086$.

31. Yu J, Chen $Y$, Chen $L$, et al. Cirmtuzumab inhibits ibrutinib-resistant, Wnt5a-induced Rac1 activation and proliferation in mantle cell lymphoma. Oncotarget 2018 ; 9(37):24731-6. doi: 10.18632/oncotarget. 25340

32. Yu J, Chen L, Chen $Y$, et al. Wnt5a induces ROR1 to associate with 14-3-3 for enhanced chemotaxis and proliferation of chronic lymphocytic leukemia cells. Leukemia 2017; 31(12):2608-14. doi: 10.1038/ leu.2017.132.

33. Prasad $\mathrm{CP}$, Södergren $\mathrm{K}$, Andersson T. Reduced production and uptake of lactate are essential for the ability of WNT5A signaling to inhibit breast cancer cell migration and invasion. Oncotarget 2017; 8(42): 71471-88. doi: 10.18632/oncotarget.17277.

34. Li J, Ying J, Fan Y, et al. WNT5A antagonizes WNT/ $\beta$-catenin signaling and is frequently silenced by promoter CpG methylation in esophageal squamous cell carcinoma. Cancer Biol Ther 2010; 10(6):617-24. doi: 10.4161/cbt.10.6.12609.

35. Ann EJ, Kim HY, Seo MS, et al. Wnt5a controls Notch1 signaling through CaMKII-mediated degradation of the SMRT corepressor protein. J Biol Chem 2012; 287(44):36814-29. doi: 10.1074/jbc.M112.356048.

36. R John M, Madeline $H$. Wnt5a secretion stimulated by the extracellular calcium-sensing receptor inhibits defective Wnt signaling in colon cancer cells. Am J Physiol Gastrointest Liver Physiol 2007; 293(1):G40311. doi: 10.1152/ajpgi.00119.2007.

37. Voloshanenko O, Schwartz U, Kranz D, et al. $\beta$-catenin-independent regulation of Wnt target genes by RoR2 and ATF2/ATF4 in colon cancer cells. Sci Rep 2018; 8(1):3178. doi: 10.1038/s41598-018-20641-5.

38. Bergenfelz C, Medrek C, Ekström E, et al. Wnt5a induces a tolerogenic phenotype of macrophages in sepsis and breast cancer patients. J Immunol 2012; 188(11):5448-58. doi: 10.4049/jimmunol.1103378.

39. Zhang W, Yan Y, Gu M, et al. High expression levels of
Wnt5a and Ror2 in laryngeal squamous cell carcinoma are associated with poor prognosis. Oncol letters 2017; 14(2):2232-8. doi: 10.3892/ol.2017.6386.

40. Dai B, Yan T, Zhang A. ROR2 receptor promotes the migration of osteosarcoma cells in response to Wnt5a. Cancer Cell Int 2017; 17:112. doi: 10.1186/s12935017-0482-y.

41. Oishi I, Suzuki H, Onishi N, et al. The receptor tyrosine kinase Ror2 is involved in non-canonical Wnt5a/ JNK signaling pathway. Genes Cells 2003; 8(7):64554. doi: 10.1046/j.1365-2443.2003.00662.x.

42. Nam S, Chung JW, Yang JY. WNT5A correlates with clinicopathological characteristics in gastric cancer: a meta-analysis. Cell Physiol Biochem 2017; 41(1):3340. doi: 10.1159/000455934.

43. Ara $\mathrm{H}$, Takagishi $\mathrm{M}$, Enomoto $A$, et al. Role for Daple in non-canonical Wht signaling during gastric cancer invasion and metastasis. Cancer Sci 2016; 107(2):1339. doi: $10.1111 /$ cas.12848.

44. Liu J, Zhang Y, Xu R, et al. PI3K/Akt-dependent phosphorylation of GSK3 $\beta$ and activation of RhoA regulate Wnt5a-induced gastric cancer cell migration. Cell Signal 2013; 25(2):447-56. doi: $10.1016 / j$.cellsig.2012.10.012.

45. Hanaki $H$, Yamamoto $H$, Sakane $H$, et al. An anti-Wnt5a antibody suppresses metastasis of gastric cancer cells in vivo by inhibiting receptor-mediated endocytosis. Mol Cancer Ther 2012; 11(2):298-307. doi: 10.1158/1535-7163.mct-11-0682.

46. Yamamoto $H$, Kitadai $Y$, Naohide Oue, et al. Laminin Y2 Mediates Wnt5a-Induced Invasion of Gastric Cancer Cells. Gastroenterology 2009; 137(1):242-52. doi: 10.1053/j.gastro.2009.02.003.

47. Kanzawa M, Semba S, Hara S, et al. WNT5A is a key regulator of the epithelial-mesenchymal transition and cancer stem cell properties in human gastric carcinoma cells. Pathobiology 2013; 80(5):235-44. doi: $10.1159 / 000346843$.

48. Zhang Y, Du J, Zheng J, et al. EGF-reduced Wnt5a transcription induces epithelial-mesenchymal transition via Arf6-ERK signaling in gastric cancer cells. Oncotarget 2015; 6:7244-61. doi: 10.18632/oncotarget. 3133 .

49. Takiguchi G, Nishita M, Kurita K, et al. Wnt5a-Ror2 signaling in mesenchymal stem cells promotes proliferation of gastric cancer cells by activating CXCL16-CXCR6 axis. Cancer Sci 2016; 107(9):290-7. doi: $10.1111 /$ cas. 12871

50. Dejmek J, Dejmek A, Säfholm A, et al. Wnt-5a protein 
expression in primary Dukes B colon cancers identifies a subgroup of patients with good prognosis. Cancer Res 2005; 65:9142-6. doi: 10.1158/0008-5472.CAN05-1710.

51. Tao J, Shi L, Huang $L$, et al. EZH2 is involved in silencing of WNT5A during epithelial-mesenchymal transition of colon cancer cell line. J Cancer Res Clin Oncol 2017; 143(11):2211-9. doi: 10.1007/s00432-017-2479-2.

52. Mehdawi LM, Prasad CP, Ehrnström R, et al. Non-canonical WNT5A signaling up-regulates the expression of the tumor suppressor 15-PGDH and induces differentiation of colon cancer cells. Mol Oncol 2016; 10:1415-29. doi: 10.1016/j.molonc.2016.07.011.

53. Cheng R, Sun B, Liu Z, et al. Wnt5a suppresses coIon cancer by inhibiting cell proliferation and epithelial-mesenchymal transition. J Cell Physiol 2014; 229(12):1908-17. doi: 10.1002/jcp.24566.

54. Wang J, Wang $X$, Liu $F$, et al. microRNA-335 inhibits colorectal cancer HCT116 cells growth and epithelialmesenchymal transition (EMT) process by targeting Twist1. Pharmazie 2017; 72(8):475-81. doi: 10.1691/ ph.2017.7489.

55. Bakker ERM, Das AM, Helvensteijn W, et al. Wnt5a promotes human colon cancer cell migration and invasion but does not augment intestinal tumorigenesis in Apc 1638N mice. Carcinogenesis 2013; 34(11):262938. doi: 10.1093/carcin/bgt215.

56. Huang TC, Lee PT, Wu MH, et al. Distinct roles and differential expression levels of Wnt5a mRNA isoforms in colorectal cancer cells. PloS One 2017; 12(8): e0181034. doi: 10.1371/journal.pone.0181034.

57. Antonia SJ, Villegas A, Daniel D, et al. Durvalumab after chemoradiotherapy in stage III non-small-cell lung cancer. N Engl J Med 2017; 377(20):1919-29. doi: 10.1056 /nejmoa1709937.

58. Liu M, Zhang Y, Zhang J, et al. MicroRNA-1253 suppresses cell proliferation and invasion of non-small-cell lung carcinoma by targeting WNT5A. Cell Death Dis 2018; 9(2):189. doi: 10.1038/s41419-017-0218-x.

59. Wang B, Tang Z, Gong H, et al. Wnt5a promotes epithelial-to-mesenchymal transition and metastasis in non-small-cell lung cancer. Biosci Rep 2017; 37(6): BSR20171092. doi: 10.1042/BSR20171092.

60. Huang $\mathrm{Cl}$, Liu D, Nakano J, et al. Wnt5a expression is associated with the tumor proliferation and the stromal vascular endothelial growth factor: An expression in non-small-cell lung cancer. J Clinc Oncol 2005; 23(34):8765-73. doi: 10.1200/jco.2005.02.2871.

61. Whang YM, Jo $U$, Sung JS, et al. Wnt5a is associated with cigarette smoke-related lung carcinogenesis via protein kinase C. PLoS One 2013; 8(1):e53012. doi: 10.1371/journal.pone.0053012.

62. Yang J, Zhang $\mathrm{K}, \mathrm{Wu}$ J, et al. Wnt5a increases properties of lung cancer stem cells and resistance to cisplatin through activation of Wnt5a/PKC signaling pathway. Stem Cells Int 2016; 2016:1690896. doi: 10.1155/2016/1690896.

63. Kobayashi Y, Kadoya T, Amioka A, et al. Wnt5a-induced cell migration is associated with the aggressiveness of estrogen receptor-positive breast cancer. Oncotarget 2018; 9(30):20979-92. doi: 10.18632/ oncotarget. 24761.

64. Prasad $C P$, Chaurasiya SK, Guilmain W, et al. WNT5A signaling impairs breast cancer cell migration and invasion via mechanisms independent of the epithelial-mesenchymal transition. J Exp Clin Cancer Res 2016; 35(1):144. doi: 10.1186/s13046-016-0421-0.

65. Prasad CP, Chaurasiya SK, Axelsson L, et al. WNT-5A triggers $\mathrm{Cdc} 42$ activation leading to an ERK1/2 dependent decrease in MMP9 activity and invasive migration of breast cancer cells. Mol Oncol 2013; 7(5):870-83. doi: 10.1016/j.molonc.2013.04.005.

66. Chrysanthou E, Gorringe KL, Joseph C, et al. Phenotypic characterisation of breast cancer: the role of CDC42. Breast Cancer Res Treat 2017; 164(2):31725. doi: 10.1007/s10549-017-4267-8.

67. Jiang W, Crossman DK, Mitchell EH, et al. WNT5A inhibits metastasis and alters splicing of $\mathrm{Cd} 44$ in breast cancer cells. PLoS One 2013; 8(3):e58329. doi: 10.1371/journal.pone.0058329.

68. Han B, Zhou B, Qu Y, et al. FOXC1-induced non-canonical WNT5A-MMP7 signaling regulates invasiveness in triple-negative breast cancer. Oncogene 2018; 37(10):1399-408. doi: 10.1038/s41388-017-0021-2.

69. Zhu Y, Tian Y, Du J, et al. Dvl2-dependent activation of Daam1 and RhoA regulates Wnt5a-induced breast cancer cell migration. PLoS One 2012; 7(5):e37823. doi: 10.1371/journal.pone.0037823.

70. Zoico E, Darra E, Rizzatti V, et al. Adipocytes WNT5a mediated dedifferentiation: a possible target in pancreatic cancer microenvironment. Oncotarget 2016; 7(15):20223-35. doi: 10.18632/oncotarget.7936.

71. Bo H, Gao L, Chen Y, et al. Upregulation of the expression of Wnt5a promotes the proliferation of pancreatic cancer cells in vitro and in a nude mouse model. Mol Med Rep 2016; 13(2):1163-71. doi: 10.3892/ mmr.2015.4642.

72. Bo H, Zhang S, Gao L, et al. Upregulation of Wnt5a 
promotes epithelial-to-mesenchymal transition and metastasis of pancreatic cancer cells. BMC Cancer 2013; 13:496. doi: 10.1186/1471-2407-13-496.

73. Wei W, Li $\mathrm{H}$, Li N, et al. WNT5A/JNK signaling regulates pancreatic cancer cells migration by phosphorylating paxillin. Pancreatology 2013; 13(4):384-92. doi: 10.1016/j.pan.2013.05.008.

74. Wei W, Sun HH, Li N, et al. WNT5A modulates cell cycle progression and contributes to the chemoresistance in pancreatic cancer cells. Hepatobiliary Pancreat Dis Int 2014; 13(5):529-38. doi: 10.1016/S14993872(14)60277-0.

75. Yamamoto $H$, Oue $N$, Sato $A$, et al. Wnt5a signaling is involved in the aggressiveness of prostate cancer and expression of metalloproteinase. Oncogene 2010; 29(14):2036-46. doi: 10.1038/onc.2009.496.

76. Thiele S, Zimmer A, Göbel A, et al. Role of WNT5A receptors FZD5 and RYK in prostate cancer cells. Oncotarget 2018; 9(43):27293-304. doi: 10.18632/oncotarget. 25551.

77. Lee GT, Kang DI, Ha YS, et al. Prostate cancer bone metastases acquire resistance to androgen deprivation via WNT5A-mediated BMP-6 induction. Br J Cancer 2014; 110:1634-44. doi: 10.1038/bjc.2014.23.

78. Lee GT, Kwon SJ, Kim J, et al. WNT5A induces castration-resistant prostate cancer via $\mathrm{CCL} 2$ and tumour-infiltrating macrophages. $\mathrm{Br} \mathrm{J}$ Cancer 2018; 118(5):670-8. doi: 10.1038/bjc.2017.451.

79. Jin F, Qu X, Fan Q, et al. Regulation of prostate cancer cell migration toward bone marrow stromal cell-conditioned medium by Wnt5a signaling. Mol Med Rep 2013; 8(5):1486-92. doi: 10.3892/mmr.2013.1698.

80. Thiele S, Göbel A, Rachner TD, et al. WNT5A has anti-prostate cancer effects in vitro and reduces tumor growth in the skeleton in vivo. J Bone Miner Res 2015; 30(3):471-80. doi: 10.1002/jbmr.2362.

81. Canesin G, Evans-Axelsson S, Hellsten R, et al. Treatment with the WNT5A-mimicking peptide Foxy-5 effectively reduces the metastatic spread of WNT5A-low prostate cancer cells in an orthotopic mouse model. PLoS One 2017; 12(9):e0184418. doi: 10.1371/journal.pone. 0184418 .
82. Ndoye A, Budina A, Webster M, et al. Role of autophagy in Wnt5A-mediated melanoma invasion and metastasis. Cancer Res 2015; 75(15):2906. doi: 10.1158/1538-7445.AM2015-2906.

83. Weeraratna AT, Jiang $Y$, Hostetter $G$, et al. Wnt5a signaling directly affects cell motility and invasion of metastatic melanoma. Cancer Cell 2002; 1(3):27988. doi: 10.1016/S1535-6108(02)00045-4.

84. Ekström EJ, Bergenfelz C, von Bülow V, et al. WNT5A induces release of exosomes containing pro-angiogenic and immunosuppressive factors from malignant melanoma cells. Mol Cancer 2014; 13:88. doi: 10.1186/1476-4598-13-88.

85. Anastas JN, Kulikauskas RM, Tamir T, et al. WNT5A enhances resistance of melanoma cells to targeted BRAF inhibitors. J Clin Invest 2014; 124(7):2877-90. doi: $10.1172 /$ JCI70156.

86. Ying J, Li H, Chen YW, et al. WNT5A is epigenetically silenced in hematologic malignancies and inhibits leukemia cell growth as a tumor suppressor. Blood 2007; 110(12):4130-1. doi: 10.1182/blood-2007-06-094870.

87. Hasan MK, Yu J, Chen L, et al. Wnt5a induces ROR1 to complex with HS1 to enhance migration of chronic lymphocytic leukemia cells. Leukemia 2017; 31(12): 2615-22. doi: 10.1038/leu.2017.133.

88. Despeaux M, Chicanne G, Rouer E, et al. Focal adhesion kinase splice variants maintain primitive acute myeloid leukemia cells through altered Wnt signaling. Stem Cells 2012; 30(8):1597-610. doi: 10.1002/ stem.1157.

89. Yu J, Chen L, Cui B, et al. Wnt5a induces ROR1/ROR2 heterooligomerization to enhance leukemia chemotaxis and proliferation. J Clin Invest 2016; 126(2):58598. doi: 10.1172/JCI83535.

90. Ng ÖH, Fırtına S, Can İ, et al. A possible role for WNT5A hypermethylation in pediatric acute lymphoblastic leukemia. Turk J Haematol 2015; 32(2):127-35. doi: 10.4274/Tjh.2013.0296.

91. Zhou HR, Fu HY, Wu DS, et al. Relationship between epigenetic changes in Wnt antagonists and acute leukemia. Oncology Rep 2017; 37(5):2663-71. doi: 10.3892/or.2017.5509. 